Title: Changing trends in young people's food behaviour and wellbeing in England in relation to family affluence between 2005 and 2014

Authors: Antonia Simon*1a ${ }^{*}$ Charlie Owen ${ }^{\mathrm{b}}$, Rebecca O'Connell ${ }^{\mathrm{b}}$ and Fiona Brooks c

${ }^{a}$ Social Science Research Unit, Department of Social Science, UCL Institute of Education, London.

${ }^{\mathrm{b}}$ Thomas Coram Research Unit, Department of Social Science, UCL Institute of Education, London.

c Faculty of Health, University of Technology, Sydney UTS, Australia.

Corresponding author: Antonia Simon, Research Officer, UCL Institute of Education, Department of Social Science, University College London, 20 Bedford Way, London, WC1H 0AL. E-mail: a.simon@ucl.ac.uk, Tel: +44 (0)20 7612 6250, Fax: +44 (0)20 76126927

This paper cannot be quoted or referred to without permission from the authors.

Full publication citation details:

1. Changing trends in young people's food behaviour and wellbeing in England in relation to family affluence between 2005 and 2014

Antonia Simon, Charlie Owen, Rebecca O'Connell \& Fiona Brooks

https://doi.org/10.1080/13676261.2017.1406599

*Corresponding author: a.simon@ucl.ac.uk 


\title{
Changing Trends in young people's food behaviour and wellbeing in England in relation to family affluence between 2005 and 2014
}

\begin{abstract}
Research shows that healthy eating improves outcomes for children and that inequalities in diet are socially determined. However, little is known about how associations between household income and the diet intake of children and young people change over time. Descriptive analysis was carried out using the Health Behaviour in School-Aged Children survey data for England for the years 2005, 2009 and 2014 to examine how breakfast, fruit, vegetable and soft drink consumption compares for young people aged 11-15 years by family affluence (low versus medium/high), gender and wellbeing measures. The results show young people in the low FAS group generally reporting less healthy eating behaviours, and differences by gender such as more soft/sugary drink consumption and lower consumption of fruit and vegetables for boys. Young people in the low FAS group also tend to have lower self-reported ratings on other wellbeing measures examined. There is evidence of a 'closing gap' between the FAS groups over time in terms of some behaviours. The patterns reported here are complex to interpret but do highlight some potentially positive effects of policies for addressing poor diets and dietary inequalities as well as some concerns given the UK context of continuing political uncertainty.
\end{abstract}

Key words: Adolescence; Health; Fruit; Vegetables; HBSC. 


\section{Introduction}

Long-standing social inequalities in health and nutrition exist in the UK (Dowler and Spencer 2007; Dowler 2008). In addition, the global economic recession, rising price of some foods (from 2007 to 2012) and so-called 'austerity' policies resulting in falling or stagnant incomes have made food less affordable for UK households in recent years (O'Connell and Hamilton 2017). However there is a lack of systematic evidence about how changing social policies and reduced affordability of food impact upon children's and young people's diet intakes. Using repeat cross sectional data from the Health Behaviour in School-aged Children (HBSC) study (Currie et al 2012), this paper adds to what is known by analysing the association between family affluence and self-reported consumption of breakfast, fruit, vegetables and soft drinks by young people aged 11-15 years in England in 2005, 2009 and 2014.

\section{Background}

Food is a source of nutrients and a medium for social relations with important health and participation dimensions, especially for young people. Social scientific approaches have demonstrated that children and teenagers seek to establish and express their identity and independence, including through the foods they consume, in an increasingly wide range of settings (Hamilton and Wills 2017; O’Connell and Brannen 2016; Wills et al 2005; Rawlins 2009). Lifecourse approaches also suggest that adolescence is a critical life stage during which many biological, physical, emotional, cognitive, behavioural and social changes take place (WHO 2006) and in which long-term health behaviours are established (Hagell, Coleman and Brooks 2013). Since growth during adolescence is faster than at any other time in an individual's life except for the first year, it is also recognised as a period of increased nutritional requirements (WHO 2005). Whilst 'healthy eating' contributes to young people's wellbeing (Shepherd et al 2006), sub-optimal diet and food practices, such as skipping breakfast (Hoyland, Dye and Lawton 2009), are associated with poor cognition and lower academic achievement as children's ability to concentrate is damaged by insufficient food or food of poor nutritious value (Dowler 2014).

The UK government recommends that boys and girls aged 11 to 18 years should consume at least five portions of a variety of fruit and vegetables per day and no more than 11 per cent food energy consumption in the form of non-milk extrinsic sugars (NMES) for those aged five years and over (PHE 2014b). However evidence from the National Diet and Nutrition Survey (NDNS) suggests that these targets are not being met (Bates et al 2011). For 
example NDNS data for 2008 to 2012 shows mean consumption of fruit and vegetables for children aged 11 to 18 years was 3.0 portions per day for boys and 2.7 portions per day for girls and only 10 per cent of boys and 7 per cent of girls in this age group were found to have met the five-a-day recommendation for children aged 11 to 18 years (PHE 2014b). Sugary drinks are known to contribute to the higher than recommended intakes of NMES, with 11-18 year olds having the highest reported mean consumption of soft drinks (PHE 2015): 78 per cent of this age group consumed 'soft drinks' such as sugar-sweetened beverages (SSB) over a four-day period (PHE 2014a).

There are also known differences between adolescents by household income that appear to reflect a broader social gradient in health and diet intake. There is evidence that inequalities of food and diet in the UK are socially determined (Dowler 2008). Analysis of the NDNS by equivalised income (a measure that takes account of the differences in a household's size and composition) shows differences in diet and nutrient intake, specifically that those in lower income quintiles tend to have poorer diets, particularly with respect to fruit and vegetable consumption (PHE 2014b). Mean fruit and vegetable consumption expressed in five-a-day portions was found to be significantly lower in all age/sex groups in income quintile 1 (lowest income) compared with quintile 5 (highest income) (ibid). Reflecting household level data, the national report for the HBSC study in England for 2009 found that 'family affluence' is an important predictor of young people's health, with higher affluence associated with higher reported fruit intake and greater likelihood of daily breakfast consumption (Currie et al 2012). Diet is also reported to vary by gender, with for example boys more likely to consume breakfast than girls (ibid).

In the context of economic austerity and rising food prices there is evidence that healthier foods have become more expensive than unhealthier ones (Jones et al 2012) and, at the household level, research suggests that reduced affordability of food has impacted on the purchase of healthy foods. For example, the Department for Environment, Food and Rural Affairs' (Defra) annual analysis of the Living Costs and Food Survey (food purchase data), shows that UK household purchases of fruit and vegetables were 11.4 per cent lower in 2014 than their peak in 2006, with low income group households purchasing the least amount of fruit and veg: 3.0 portions per person/ day (Defra 2015). However less is known about how economic phenomena and policy changes, such as austerity and rising food prices, impact upon the diet or nutrition intakes of young people.

In this paper, we seek to extend and complement the analysis by Currie et al (2012) described above by examining in detail trends for England using the HBSC data for 2005, 
2009, and 2014, addressing the research question: 'What is the relationship between family affluence and self-reported consumption of breakfast, fruit, vegetables and soft drinks by young people aged 11-15 years in England and how does this change over time?' We also examine the relationship between self-reported food behaviours, gender and wellbeing. Given the UK context of continuing political uncertainty, economic austerity, higher inflation including food prices, and sustained policy interest in improving the diet intake of children and young people, the analysis is very timely.

\section{Method}

\section{Secondary data and study participants}

The analysis reported in this paper was conducted on data from the HBSC study for England in 2005, 2009 and 2014. The HBSC is a multi-national, cross sectional survey that uses a school-based self-completion questionnaire to collect data every four years from 11-, 13- and 15-year-old boys and girls about their health and well-being, social environments and health behaviours. The HBSC now includes 44 countries and regions across Europe and North America. Some core questions are asked in all of the countries (the "mandatory questionnaire') whilst additional batteries of questions ('optional modules') are asked by groups of countries with particular shared interests. Some questions are country specific ('country-level only'). In part this reflects the funding arrangements of the study which are such that each survey is funded nationally and funders may require that particular questions are included. Further information about the HBSC can be found at: http://www.hbsc.org/about/.

\section{Measures and analysis}

Currie et al (2008a; 2008b) developed a Family Affluence Scale (FAS) for the HBSC. It consisted of four questionnaire items concerning family ownership of a car (scored: No 0, Yes, one 1, Yes, two or more 2), child having their own bedroom (scored No 0, Yes 1), having family holidays (scored: Not at all 0 , Once 1 , Twice 2 ) and family computers (scored: None 0 , One 1, Two 2, More than two 3). The scale ranged from zero to eight. The scale was then coded into three categories: low (0-3), medium (4-6) and high (7-8). This three-point scale has been used here. For the analysis reported here, we compared 'low FAS' with 'higher FAS' (combining medium and high). Distribution of values for each of the years did not distinguish much between medium and high FAS so we felt justified in merging these two categories. The way we have categorised these variables follows analysis reported by 
Niclasen et al (2013) who examined the adverse health effects of experiencing food insecurity among Greenlandic school children.

HBSC England includes a number of variables related to food behaviours that may be used as indicators of healthiness of diet in relation to recommended intake. Descriptive analysis (using SPSS) was carried out for the following food behaviours in relation to FAS: Eats Breakfast 5-6 times a week or more, Eats Vegetables 5-6 times a week or more, Eats Fruit 5-6 times a week or more, and drinking soft drinks 5-6 times a week or more. Three measures of general wellbeing are provided in the HBSC dataset: self-rated health good or excellent, self-reported 'very best' quality of life and self-reported academic achievement as 'very good/good'. These are the only measures of 'wellbeing' (wellbeing in the broadest sense) provided in the HBSC and are reported in the HBSC international reports as indicators of general wellbeing for young people (Currie et al 2012). Previously, analysis of the HBSC has discussed how important, especially during adolescence, it is to include life satisfaction (which is an important cognitive aspect of well-being) and not just report the "healthoriented" satisfaction with life, i.e., the child's/adolescent's current situation (Suldo et al 2006). We examined these same 'wellbeing' measures in relation to FAS. Finally, food behaviours and the wellbeing measures were examined by gender. $\mathrm{P}$ values for the Pearson chi-square are presented along with phi coefficients (measuring the strength of relationship: Cohen 1977, 223).

The distribution of these variables for each of the HBSC data time points analysed (2005, 2009 and 2014) are given in table one below. This table shows that the proportion of young people in the low FAS group was lowest in 2014 (7.4\% in 2005, 11.4\% in 2009 and $6.3 \%$ in 2014), indicating an improvement in socio-economic circumstances for young people over time. Although the FAS has been shown to have acceptable validity and reliability (e.g. Currie et al 1997, Kehoe and Hare 2010), Schnohr et al (2008) highlighted its limitations for cross-country comparisons due to differential response on the individual items. However, that is not relevant here as we are only analysing data for one country. More recently, Schnohr et al (2013) have identified a drift in item scores over time: in particular, a big growth in computer ownership in the 2009/10 survey produced an increasing mean for the scale, potentially reducing its validity. In the current study we also found a growth in computer ownership, giving an increase in mean FAS: in 2005 36.5\% of households had more than two computers, by 2014 this had increased to $71.6 \%$.

The percentages for the health and wellbeing measures used and for FAS, for each of the three surveys, are shown in Table 1 . There is a slightly higher proportion of girls in the 
low FAS group at each time point but the gap between boys and girls narrows over time and is smallest in 2014.

\section{TABLE ONE HERE.}

\section{Results}

Overall for young people aged 11-15 years the proportion eating breakfast and vegetables 5-6 times per week or more has remained stable over time (for breakfast, the proportion changes from $65 \%$ in 2005 to $61 \%$ in 2009 to $66 \%$ in 2014 and for vegetables the proportion changes from $63 \%$ in 2005 to $59 \%$ in 2009 to $63 \%$ in 2014: see Table 4). However, there has been a decline in the proportion reporting drinking soft drinks five or more times a week over time (from 34\% in 2005 to $52 \%$ in 2009 to $22 \%$ in 2014: see table one). Examining these trends comparing low FAS and higher FAS young people produces a more complex picture. These results are discussed in the sections below.

\section{Breakfast consumption}

The proportion of young people aged 11-15 years eating breakfast five to six times per week or more declined in 2009 compared with 2005, but then rose between 2009 and 2014 so that the proportion of young people consuming breakfast at least five times per week was higher in 2014 than in 2005 (from $54.7 \%$ in 2005 to $65.9 \%$ in 2014 for all young people aged 11-15 years: Table 1). Most of the rise in breakfast consumption between 2005 and 2014 was driven by the increase in the proportion of young people in the low FAS group eating breakfast (a rise from $50.2 \%$ in 2005 to $54.7 \%$ in 2014: Table 2).

\section{(TABLE TWO)}

\section{Consumption of fruit and vegetables}

The proportion of young people eating fruit five to six times per week or more declined overall in 2009 compared with 2005, and then in 2014 was at about the same level as 2009. The pattern is different when comparing the low FAS and higher FAS groups however (see Table 2). Fruit consumption five to six times per week or more declined steadily for the higher FAS group over the three time points (from 59.9\% in 2005 to 54.7 in 2009 to 53.4\% in 2014: Table 3). In contrast, for the low FAS group, the proportion of young people eating fruit five to six times per week or more declined between 2005 and 2009, 
before rising again in 2014 to a higher proportion than in 2005 (declining from $44.9 \%$ in 2005 to 40.8 in 2009 before rising to $45.8 \%$ in 2014: Table 3). Although a greater proportion of the higher FAS group reported eating fruit 5-6 times per week or more in 2014 compared with the low FAS group, the gap between the two groups had narrowed when compared to the respective proportions for these groups in 2005 (a gap of 7 percentage points in 2014 compared with 15 in 2005). For young people aged 11-15 years, almost the same proportion ate vegetables five to six times per week or more in 2005 and 2014, with a slight dip in 2009 (Table 4). This pattern was true both for the higher FAS groups and for the low FAS group (the gap between the two groups was 15.9 percentage points in 2014: Table 4).

(TABLE THREE)

\section{(TABLE FOUR)}

\section{Consumption of 'soft drinks'}

The HBSC question examined on soft drinks is a measure of the consumption of drinks that contain sugar, such as coke, squash or other soft drink. Compared to 2005 and 2009 there was a decline overall in 2014 in the proportion of young people reporting that they consumed soft drinks five to six times per week (Table 5). However this decline in soft drink consumption followed a sharp rise in 2009 for both groups. In 2009 similar proportions of young people in the low FAS and higher FAS groups said that they drank soft drinks five to six times per week or more. The pattern observed in 2009 of higher soft drink consumption is different to that found in 2005, when a third more young people in the low FAS group reported drinking soft drinks five to six times per week or more. There was almost no difference in 2014 between the low and higher FAS groups for this variable. Whilst smaller proportions of young people from both groups reported drinking soft drinks five to six times per week in 2014, the most dramatic difference since 2005 is in the low FAS group. The observed reduction in soft drink consumption between 2009 and 2014 for all young people could be related to a change in wording about sugary drinks in the HBSC England questionnaire (Brooks et al 2015; Brooks et al 2011, the protocol can be requested from the HBSC data hub). In 2009, the wording for the HBSC question on sugary drinks was: "How many times a week do you usually drink coke, squash or other soft drinks that contain sugar?". In 2014, the question was changed to: "How many times a week do you usually drink fizzy drinks that contain sugar e.g. Coke, Pepsi, and Lemonade. NOT diet drinks?". The 
change in question wording between 2005/9 and 2014, especially the addition of the phrase "NOT DIET DRINKS" means the two questions are not asking exactly the same thing over time and are likely to have been interpreted differently by the young people questioned at each time point. Also, in 2014 in the HBSC survey for England, a further two questions were included: "How many times a week do you usually drink energy drinks e.g. Red Bull and Monster?" and "How many times a week do you usually drink squash that contains sugar"?. This means that not only has the question wording changed between 2005/9 to 2014 but that two components of the earlier question have been separated out in 2014. These changes to the question wording in 2009 and 2014 could mean the question was understood differently by young people at each of these time points, making it complicated to interpret whether the reduction we observed in soft drinks between 2009 and 2014 is real or an artefact of these wording changes within the HBSC. However, while the wording changed for all groups, the differences over time were not the same for the FAS groups. We observed that the low FAS group saw the largest decline in consumption of soft drinks over time. It is unlikely therefore that the wording changes alone to soft drinks in the HBSC explain all of the difference observed between the low and higher FAS groups between 2009 and 2014.

\section{(TABLE FIVE)}

\section{Other measures of wellbeing}

Three aspects of wellbeing reported by study participants: 'self-rated health as good or excellent', 'very best quality of life' and 'good or very good academic achievement' were also related to FAS. There is a clear association overall between 'healthy food behaviours' (all the healthy eating food variables except for vegetables) and all three aspects of wellbeing we examined (Tables 6 to 8). This pattern holds across time. However, there were some notable differences between the low FAS and higher FAS groups: a higher proportion of the higher FAS groups reported having good or very good academic achievement at each time point (Table 5). This pattern was also true for self-rated health for the years 2005 and 2009. However, in 2014 a greater proportion of the low FAS group reported positive ratings of quality of life compared with the higher FAS groups. 


\section{Gender}

Eating behaviours also varied by gender. In particular, a higher proportion of boys aged 11-15 years reported consuming breakfast 5-6 times per week or more (71\% in 2005, $66 \%$ in 2009 and $74 \%$ in 2014) compared with girls (59\% in 2005, 55\% in 2009 and 59\% in 2014). This difference is much bigger than any of the other difference between boys and girls. However, a higher proportion of girls consumed fruit (e.g. 55\% compared with $50 \%$ of boys in 2014). A lower proportion of both boys and girls consumed fruit than vegetables over time (comparing 2005 with 2014). Boys were also more likely to drink soft drinks (38\% in $2005,56 \%$ in 2009 and $25 \%$ in 2014) as compared with girls (30\% in 2005, 50\% in 2009 and $20 \%$ in 2014) at each time point. There was a reduction in 2014 for both boys and girls, but, as noted above, there was a change in the wording for the question which may have influenced the responses.

Gender differences were also observed for the three aspects of wellbeing examined. A higher proportion of boys than girls aged 11-15 reported having good or excellent self-rated health at each time point (e.g. $88 \%$ compared with $83 \%$ in 2014). Conversely a greater proportion of girls than boys reported having self-rated good or very good academic achievement at each time point, and the gap between the two groups increases over time (70\% of boys and $74 \%$ of girls in $2005,71 \%$ of boys and $76 \%$ of girls in 2009 , and $71 \%$ of boys and $81 \%$ of girls in 2014). The pattern for self-rated quality of life changes over time, being the same proportion for both groups in 2005, and higher for boys in 2009 (12\% compared with 9\% girls) and in 2014 (12\% compared with 10\% of girls), which supports findings reported elsewhere of perceived quality of life being higher for boys than girls (Jiménez-Iglesias et al 2015).

\section{Discussion}

Descriptive analysis was carried out on variables related to young people's selfreported food and eating behaviours in the Health Behaviour in School-Aged Children (HBSC) survey data for England for the years 2005, 2009 and 2014. The analysis finds a small rise over time in the proportion of young people aged 11-15 years consuming breakfast, vegetables and fruit five to six times per week and a reduction in the proportion drinking soft drinks every day. Reflecting analysis by Currie et al (2012) the patterns also vary by FAS for example, daily breakfast consumption is less common in families with lower affluence. The largest changes over time reported here appear to be for the low FAS group in their increased reported consumption of breakfast and reduction in sugary drinks. Overall we find 
there's no pattern in the strength of relationship over time with each of our food and wellbeing measures. These results are discussed in more detail below, in relation to changing UK economic and policy contexts.

The analysis finds a big gender difference in the consumption of breakfast, with boys much more likely to report eating breakfast than girls. This difference was consistent across the three surveys. Overall there was an increase over time in the consumption of breakfast by young people aged 11-15 years, and that was higher at each time point for the higher FAS groups compared to the low FAS group. However, what is particularly interesting is that it is the low FAS group that has seen the largest improvement over time. This could be because of changes made to the provision of school breakfasts. The School Food Plan launched in 2013 (Dimbleby and Vincent 2013) includes an Action that sets out a Department for Education commitment to set up financially self-sufficient breakfast clubs to increase healthy breakfast provision for children who are arriving at school hungry. Whilst there is a lack of research on breakfast club provision in the UK, a report by cereal manufacturer Kellogg's suggests the use of breakfast clubs has increased by 45 per cent since 2008 (Kellogg's 2014) and a proportion of these offer free breakfast to all pupils or those eligible for free school meals (FSM). Charitable support (e.g. from Kellogg's or social enterprise Magic Breakfast) may be accessed by schools with a high proportion of children eligible for free school meals. Given that many of the young people in the low FAS group are likely to be entitled to receive FSMs, this could be a possible explanation for why a greater proportion of the low FAS group consume breakfast over time and why the gap between the low FAS and higher FAS groups narrows over time.

It is also possible that the reduction observed in the proportion of young people reporting that they drink sugary drinks five to six times per week, and in particular the steep fall in the proportion of young people in the low FAS groups reporting this, perhaps reflects the success of 'behaviour change' public policy intervention. The Change4Life social marketing programme (Department of Health 2011) that was developed as part of the childhood obesity prevention strategy, launched in 2009 and originally targeted parents of children aged five to 11, 'particularly those from segments of the population where parental attitudes, beliefs and behaviours indicated that their children were most likely to gain excess weight' (ibid: 3). Whilst we cannot be confident that the reduction observed in the proportion of young people drinking soft drinks over time was not due to changes to the question wording between 2005/9 and 2014, it is unlikely that the soft drink question was interpreted differently by young people in the low and higher FAS groups. This means the reduction in 
soft drink consumption for the low FAS group is likely to be true. However, other data suggests that sugary drinks consumption for all groups remains high and has led to the UK government adopting NICE recommendations to introduce a sugar a tax or levy that will come into effect in April 2018 (HM Treasury 2016), suggesting that behaviour change strategies have not been very sucessful in reducing sugar intake. Boys were slightly more likely to drink sugary drinks than girls in all three surveys.

The findings also indicate differences between the consumption of vegetables and fruits between the low FAS and higher FAS groups. The findings show that whilst eating fruit five to six per week declined steadily for the higher FAS group over the three time points, for the low FAS group, the proportion of young people eating fruit five to six times per week declined between 2005 and 2009, before rising again in 2014 to a higher proportion than for 2005. Although a greater proportion of the higher FAS group reported eating fruit five to six times per week in 2014 compared with the low FAS group, then, the gap between the two groups had narrowed compared to 2005.

It is not straightforward to interpret these patterns. Rising food prices may explain some of the decline in reported fruit consumption between 2005 and 2009: UK food prices increased by 11.5 per cent in real terms between 2007 and 2012 (when prices peaked), and more for fresh fruit (23\%) and vegetables (24\%) (Defra 2015). Research suggests that at the household level purchase of fruits and vegetables declined over 'the great recession' (Griffith et al 2014). Analysis by Defra suggests that the effects of rising prices are greatest in households that spend a larger proportion of their budget on food and in 2012: those in the lowest income decile spent 22 per cent more on food in 2012 than in 2007 but purchased 5.7 per cent less, buying significantly fewer portions of fruit and vegetables than previously (Defra 2015). Since gradual price reductions since 2013 have reduced the real terms increase in food prices to 8 per cent compared to 2007, this may explain the levelling off observed for higher FAS groups. However, it does not explain why reported fruit consumption has risen for the lower FAS group since 2009. Some evidence (e.g. Nicklas et al 1998; Sahota et al 2001, 2001b) supports the interpretation that nutrient-based standards (now supplanted by food-based standards), which were introduced in most secondary schools in England in 2009, increase young people's consumption of fruit. However this does not account for the difference between low and higher FAS groups.

The analysis also found a positive relationship overall between healthy food behaviours (except for vegetables) and all three aspects of wellbeing examined. However, those in the low FAS group, generally rated their health and academic achievement worse 
than the higher FAS groups. Furthermore, girls rated all aspects of wellbeing, apart from academic achievement, worse than boys, despite that they generally reported healthier food behaviours, with the exception of eating breakfast regularly. These results reflect that the relationship between food, health and wellbeing for teenagers is complex and that it is gendered as well as classed. It is likely that body image plays an important role and may explain the lower proportion of girls eating breakfast (Austin and Gortmaker 2001).

There are some data limitations with using the FAS that need to be acknowledged. FAS was developed by the HBSC team as a means of differentiating poor and affluent families in national and cross-national samples. However, a number of challenges in the using this measure are cited in the HBSC protocol. The (original) FAS measures were based on absolute affluence (i.e., quantity of material assets in the family) so the FAS scores might not equally correspond to relative differences in affluence across different socioeconomic conditions (i.e. between countries or between survey years). They therefore suggest it is problematic to equate the average or distribution of FAS data or the associations between FAS and health over time or between countries (Currie et al 2008b). This could explain why we find differences in the proportion of young people in the low FAS group for each survey year examined.

\section{Conclusions}

In the UK a longstanding social gradient in health and diet intake is related to inequitable economic access to sufficient healthy food (Dowler, 2008). The analysis reported here confirms that this pattern is found among teenagers and that it persists over time. The results show marked inequalities by FAS, with young people in the low FAS group generally reporting less healthy eating behaviours than the medium/high FAS group, and differences by gender, for example, more soft/sugary drink consumption and lower consumption of fruit and vegetables for the boys. Young people in the low FAS group also tend to have lower selfreported ratings on some of the other wellbeing measures examined - health and academic achievement. However, as discussed, the patterns are more complex than this at first suggests. There is evidence of a 'closing gap' between the low FAS and higher FAS groups in terms of some behaviours, namely eating breakfast regularly, eating more fruit and consuming less sugary drinks over time. However, as noted above, the meaning of FAS over time may have changed due to differential change on the items of the scale (Currie et al 2012). 
Whilst some of the trends appear encouraging, they are difficult to interpret and not necessarily cause for celebration. For example the closing gap between higher and low FAS groups regarding fruit consumption is explained not only by a rise in fruit consumption by the low FAS group but a fall and levelling off among the higher FAS group that occurs in the context of high food - especially fruit - prices. Some of the changes, particularly the increase in the proportion of the low FAS group eating breakfast regularly over time, are more hopeful and could be related to public policy interventions that are setting out to improve children's diets and tackle socioeconomic inequalities in health. However, although more than half of girls ate breakfast, they were much less likely to do so than the boys. Whilst the relationships for patterns of diet by FAS reported here are statistically significant, the Phi coefficients reported in the tables show these relationships are weak. Nevertheless, the findings reported in this paper highlight some potentially positive effects of policies addressing poor diets and dietary inequalities as well as some cause for concern given the UK context of continuing political uncertainty, economic austerity and higher inflation including food prices that are making it more difficult for families to access diets that meet their needs for health and social participation.

\section{Funding acknowledgements}

The research leading to these results have received funding from the European Research Council under the European Union's Seventh Framework Programme (FP7/2007-2013) /ERC grant agreement $n^{\circ} 337977$.

\section{Acknowledgements}

We would like to thank the Health Behaviour in School-aged Children study for England for granting us access to the 2005, 2009 and 2014 data for the analysis reported in this paper. The HBSC web site (http://www.hbsc.org/) includes a link for downloading the open access survey data for 2005/6. We would also like to acknowledge the rest of the UK research project team, Julia Brannen, Abigail Knight, Laura Hamilton and Penny Mellor. 


\section{References}

Austin, S.B, and S.L. Gortmaker. 2001. "Dieting and smoking initiation in early adolescent girls and boys: a prospective study". American Journal of Public Health, 91(3), 446-450.

Bates, B., A. Lennox, A. Prentice, C. Bates, and G. Swan. 2011. National Diet and Nutrition Survey. Headline Results from Years 1, 2 and 3 (combined) of the Rolling Programme (2008/2009 - 2010/11). London: Department of Health.

Brooks F., J, Magnusson., E, Klemera., K, Chester., N, Spencer, and N. Smeeton. 2015. HBSC England National Report: Findings from the 2014 HBSC study for England. Hatfield: University of Hertfordshire.

Brooks F, J, Magnusson., E, Klemera., N, Spencer, and A, Morgan. 2011. HBSC England National Report. Findings from the 2010 HBSC study for England. Hatfield: University of Hertfordshire.

Cohen, J. 1977. Statistical Power Analysis for the Behavioral Sciences. New York: Academic Press.

Currie, C.E., R.A, Elton., J, Todd, and S, Platt. 1997. "Indicators of socioeconomic status for adolescents: the WHO Health Behaviour in School-aged Children Survey". Health Education Research, 12(3).

Currie, C. et al., eds. 2008a. Inequalities in Young People's Health: HBSC International Report from the 2005/06 Survey. Health Policy for Children and Adolescents, No. 5. Copenhagen: WHO Regional Office for Europe.

Currie C., M, Molcho., W, Boyce., B.E, Holstein., T, Torsheim, and M, Richter. 2008b. "Researching health inequalities in adolescents: the development of the Health Behaviour in School-aged Children (HBSC) Family Affluence Scale”. Social Science and Medicine, 2008; 66 (6): 1429-1436. 
Currie, C et al., eds. 2012. Social Determinants of Health and Well-Being Among Young People. Health Behaviour in School-aged Children (HBSC) study: International Report From the 2009/2010 Survey. Health Policy for Children and Adolescents, No. 6. Copenhagen, WHO Regional Office for Europe.

Department for Environment, Food and Rural Affairs. 2015. Food Statistics Pocketbook 2015. London: Defra.

Department of Health. 2011. Change4Life Three Year Social Marketing Strategy. London: $\mathrm{DH}$.

Dowler, E. and N, Spencer. eds. 2007. Challenging health inequalities: from Acheson to "choosing health". Bristol: Policy Press.

Dowler, E. 2008. "Food and health inequalities: the challenge for sustaining just consumption”. Local Environment, 13:8, 759-772. DOI: 10.1080/13549830802478736

Dowler, E. 2014. "Food banks and food justice in “Austerity Britain” Pp. 160-175 in: Riches, G. and Silvasti, T., eds. First World Hunger Revisited. Basingstoke: Palgrave Macmillan

Griffith, R., M, O'Connell, and K, Smith. 2013. Food Expenditure and Nutritional Quality over the Great Recession. Briefing Note 143. London: IFS.

Hagell, A., J, Coleman, and F, Brooks. 2013. Key Data on Adolescence 2013. London: Association for Young People's Health.

Hamilton, L., and W, Wills. 2017. "Patterns of sugar-sweetened beverage consumption amongst young people aged 13-15 years during the school day in Scotland". Appetite, 01.09.2017. http://dx.doi.org/10.1016/j.appet.2017.05.006

HM Treasury. 2016. Budget 2016. London: HM Treasury. 
Hoyland, A., L, Dye, and C.L, Lawton. 2009. "A systematic review of the effect of breakfast on the cognitive performance of children and adolescents". Nutr Res Rev, 22(2):220-43. doi: 10.1017/S0954422409990175.

Jiménez-Iglesias, A., C. Moreno, P. Ramos, and F. Rivera. 2015. "What Family Dimensions are Important for Health-Related Quality of Life in Adolescence?" Journal of Youth Studies, 18(1), 53-67. DOI:10.1080/13676261.2014.933191

Kehoe, S, and L, O'Hare. 2010. "The reliability and validity of the Family Affluence Scale". Effective Education, 2(2): 155-164.

Kellogg's. 2014. An Audit of School Breakfast Club Provision in the UK. A Report by Kellogg's. Manchester: Kellogg's.

Nicklas, T.A., C.C. Johnson, L. Myers, R.P. Farris, and A, Cunningham. 1998. "Outcomes of a high school program to increase fruit and vegetable consumption: Gimme $5-$ a fresh nutrition concept for students”. Journal of School Health. 68 (6): 248-253.

Niclasen, B., M. Petzold, and C.W. Schnohr. 2013. "Adverse Health Effects of Experiencing Food Insecurity Among Greenlandic School Children." International Journal of Circumpolar Health, 72: 20849. http://dx.doi.org/10.3402/ijch.v72i0.20849.

O'Connell, R. and L, Hamilton. 2017. Pp. 94-100 in V. Cooper, D. Whyte (Eds.), The Violence of Austerity. London: Pluto.

O’Connell, R. and J, Brannen. 2016. Food, Families and Work. London: Bloomsbury.

Public Health England. 2014b. National Diet and Nutrition Survey Results from Years 1, 2, 3 and 4 (combined) of the Rolling Programme (2008/2009-2011/2012) (4). London: PHE.

Public Health England. 2015. Sugar Reduction: The Evidence for Action. London: PHE.

Sahota, P., M.C, Rudolf., R, Dixey., A.J., Hill., J.H, Barth, and J, Cade. 2001a. "Evaluation of implementation and effect of primary school based intervention to reduce risk 
factors for obesity”. BMJ, 323 (7320), 1027-1029.

Sahota, P., M.C, Rudolf., R, Dixey., A.J., Hill., J.H, Barth, and J, Cade. 2001b. "Randomised controlled trial of primary school based intervention to reduce risk factors for obesity". BMJ, 323 (7320), 1029-1032.

Schnohr, C.W., S, Kreiner., E.P, Due., C, Currie., W, Boyce, and F, Diderichsen. 2008. "Differential item functioning of a Family Affluence Scale: validation study on data from HBSC 2001/02”. Social Indicators Research, 89(1): 79-95.

Schnohr, C.W., G, Makransky., S, Kreiner., T, Torsheim., F, Hofmann., B, De Clercq., F.J, Elgar, and C, Currie. 2013. "Item response drift in the Family Affluence Scale: A study on three consecutive surveys of the Health Behaviour in School-aged Children (HBSC) survey". Measurement, 46(9): 3119-3126.

Shepherd, J., A, Harden., R, Rees., G, Brunton., J, Garcia., S, Oliver, and A, Oakley. 2006. "Young people and healthy eating: a systematic review of research on barriers and facilitators". Health Education Research, 21(2): 239-257.

Rawlins, E. 2009. “Choosing Health? Exploring Children's Eating Practices at Home and at School". Antipode, 41 (5):1084-1109.

World Health Organisation. 2005. Nutrition in Adolescence: Issues and Challenges for the Health Sector: Issues in Adolescent Health and Development. (WHO discussion papers on adolescence). Geneva: World Health Organisation.

World Health Organisation. 2006. Food and Nutrition Policy for Schools. A Tool for the Development of School Nutrition Programmes in the European Region. Programme for Nutrition and Food Security. World Health Organisation: Copenhagen.

Wills, W., K, Backett-Milburn., S, Gregory, and J, Lawton. 2005. "The influence of the secondary school setting on the food practices of young teenagers from disadvantaged backgrounds in Scotland". Health Education Research, 20(4): 458-465. 
Table 1: Variables analysed in 2005, 2009 and 2014 HBSC, England: all young people

\begin{tabular}{|l|c|c|c|}
\hline & $\begin{array}{c}2005 \\
(\mathrm{n}=4223)\end{array}$ & $\begin{array}{c}2009 \\
(\mathrm{n}=3003)\end{array}$ & $\begin{array}{c}2014 \\
(\mathrm{n}=4914)\end{array}$ \\
\hline Eating breakfast on all schooldays & $64.7 \%$ & $59.9 \%$ & $66.4 \%$ \\
\hline $\begin{array}{l}\text { Eating fruit 5-6 times a week or } \\
\text { more }\end{array}$ & $58.8 \%$ & $52.7 \%$ & $52.7 \%$ \\
\hline $\begin{array}{l}\text { Eating vegetables 5-6 times a week } \\
\text { or more }\end{array}$ & $62.5 \%$ & $57.8 \%$ & $62.7 \%$ \\
\hline $\begin{array}{l}\text { Drinking soft drinks 5-6 times a } \\
\text { week or more }\end{array}$ & $34.1 \%$ & $52.6 \%$ & $22.3 \%$ \\
\hline $\begin{array}{l}\text { Self-rated health good or excellent } \\
\text { Very best quality of life }\end{array}$ & $81.4 \%$ & $83.8 \%$ & $16.6 \%$ \\
\hline $\begin{array}{l}\text { Good or very good academic } \\
\text { achievement }\end{array}$ & $72.4 \%$ & $73.8 \%$ & $75.9 \%$ \\
\hline Low FAS group only & $7.4 \%$ & $11.4 \%$ & $6.2 \%$ \\
\hline $\begin{array}{l}\text { Gender \% in low FAS group: } \\
\text { Boys } \\
\text { Girls }\end{array}$ & $8.0 \%$ & $10.4 \%$ & $12.0 \%$ \\
\hline
\end{tabular}


Table 2: Associations of 'FAS' by per cent of young people who eat breakfast for 2005, 2009 and 2014 HBSC, England

\begin{tabular}{|l|r|r|r|}
\hline \multirow{2}{*}{} & \multicolumn{3}{|c|}{ \% of young people who eat breakfast } \\
\cline { 2 - 4 } & $2005(\mathrm{n}=4223)$ & $2009(\mathrm{n}=3003)$ & $\begin{array}{r}2014 \\
(\mathrm{n}=4914)\end{array}$ \\
\hline Low FAS & $50.2 \%$ & $45.6 \%$ & $54.7 \%$ \\
\hline Medium/High FAS & $66.0 \%$ & $61.9 \%$ & $66.6 \%$ \\
\hline All & $64.9 \%$ & $60.1 \%$ & $65.9 \%$ \\
\hline Chi-square & $\mathrm{P}<.001 *$ & $\mathrm{P}<.001 *$ & $\mathrm{P}<.001 *$ \\
\hline Phi & .087 & .105 & .060 \\
\hline
\end{tabular}


Table 3: Associations of 'FAS' by per cent of young people who eat fruit 5-6 times a week or more for 2005, 2009 and 2014 HBSC, England

\begin{tabular}{|l|r|r|r|}
\hline \multirow{2}{*}{} & \multicolumn{3}{|c|}{ \% of young people who eat fruit 5-6 times a week or } \\
& \multicolumn{3}{|c|}{ more } \\
\cline { 2 - 4 } & $2005(\mathrm{n}=4223)$ & $2009(\mathrm{n}=3003)$ & $2014(\mathrm{n}=4914)$ \\
\hline Low FAS & $44.9 \%$ & $40.8 \%$ & $45.8 \%$ \\
\hline Medium/High FAS & $59.9 \%$ & $54.7 \%$ & $53.4 \%$ \\
\hline All & $58.8 \%$ & $53.1 \%$ & $52.9 \%$ \\
\hline Chi-square & $\mathrm{P}<.001^{*}$ & $\mathrm{P}<.001 *$ & $\mathrm{P}=0.014 *$ \\
\hline phi & .080 & .088 & .037 \\
\hline
\end{tabular}


Table 4: Associations of 'FAS' by per cent of young people who eat vegetables 5-6 times a week or more for 2005, 2009 and 2014 HBSC, England

\begin{tabular}{|l|r|r|r|}
\hline \multirow{2}{*}{} & \multicolumn{3}{|c|}{ \% of young people who eat vegetables 5-6 times a } \\
\cline { 2 - 4 } & $2005(\mathrm{n}=4223)$ & $2009(\mathrm{n}=3003)$ & $2014(\mathrm{n}=4914)$ \\
\hline Low FAS & $48.8 \%$ & $44.0 \%$ & $48.7 \%$ \\
\hline Medium/High FAS & $64.1 \%$ & $60.6 \%$ & $64.6 \%$ \\
\hline All & $63.0 \%$ & $58.7 \%$ & $63.6 \%$ \\
\hline Chi-square & $\mathrm{P}<.001 *$ & $\mathrm{P}<.001 *$ & $\mathrm{P}<.001 *$ \\
\hline Phi & .082 & .107 & .080 \\
\hline
\end{tabular}


Table 5: Associations of 'FAS' by per cent of young people who drink 'soft drinks' 5-6 times a week or more for 2005, 2009 and 2014 HBSC, England

\begin{tabular}{|l|r|r|r|}
\hline \multirow{2}{*}{} & \multicolumn{3}{|c|}{$\begin{array}{r}\text { \% of young people who drink 'soft drinks' 5-6 } \\
\text { times a week or more }\end{array}$} \\
\cline { 2 - 4 } & $2005(\mathrm{n}=4223)$ & $2009(\mathrm{n}=3003)$ & $2014(\mathrm{n}=4914)$ \\
\hline Low FAS & $40.3 \%$ & $53.0 \%$ & $24.1 \%$ \\
\hline Medium/High FAS & $33.3 \%$ & $52.3 \%$ & $22.2 \%$ \\
\hline All & $33.8 \%$ & $52.4 \%$ & $22.3 \%$ \\
\hline Chi-square & $\mathrm{P}=0.012 *$ & $\mathrm{P}=0.827$ & $\mathrm{P}=0.455$ \\
\hline Phi & .039 & .004 & .011 \\
\hline
\end{tabular}


Table 6: Associations of 'FAS' by per cent of young people who self-rate their health as 'good' or 'excellent' for 2005, 2009 and 2014 HBSC, England

\begin{tabular}{|l|r|r|r|}
\hline \multirow{2}{*}{} & \multicolumn{3}{|c|}{$\%$ of young people who self-rate their health as } \\
& \multicolumn{3}{|c|}{ 'good' or 'excellent' } \\
\cline { 2 - 4 } & $2005(\mathrm{n}=4223)$ & $2009(\mathrm{n}=3003)$ & $2014(\mathrm{n}=4914)$ \\
\hline Low FAS & $67.8 \%$ & $78.3 \%$ & $79.7 \%$ \\
\hline Medium/High FAS & $82.0 \%$ & $84.0 \%$ & $86.0 \%$ \\
\hline All & $81.0 \%$ & $83.4 \%$ & $85.6 \%$ \\
\hline Chi-square & $\mathrm{P}<.001 *$ & $\mathrm{P}=0.008^{*}$ & $\mathrm{P}=0.005^{*}$ \\
\hline Phi & .095 & .048 & .043 \\
\hline
\end{tabular}


Table 7: Associations of 'FAS' by per cent of young people who report having the 'very best quality of life' for 2005, 2009 and 2014 HBSC, England

\begin{tabular}{|l|r|r|r|}
\hline \multirow{2}{*}{} & \multicolumn{3}{|c|}{ \% of young people who report having the 'very best } \\
& \multicolumn{3}{|c|}{ quality of life' } \\
\cline { 2 - 4 } & $2005(\mathrm{n}=4223)$ & $2009(\mathrm{n}=3003)$ & $2014(\mathrm{n}=4914)$ \\
\hline Low FAS & $9.7 \%$ & $7.4 \%$ & $12.2 \%$ \\
\hline Medium/High FAS & $12.1 \%$ & $9.8 \%$ & $10.8 \%$ \\
\hline All & $11.9 \%$ & $9.6 \%$ & $10.8 \%$ \\
\hline Chi-square & $\mathrm{P}=0.213$ & $\mathrm{P}=0.159$ & $\mathrm{P}=0.461$ \\
\hline Phi & .019 & .026 & .011 \\
\hline
\end{tabular}


Table 8: Associations of 'FAS' by per cent of young people who report 'good' or 'very good' academic achievement for 2005, 2009 and 2014 HBSC, England

\begin{tabular}{|l|r|r|r|}
\hline \multirow{2}{*}{} & \multicolumn{3}{|c|}{$\begin{array}{r}\text { \% of young people who report 'good' or 'very } \\
\text { good' academic achievement }\end{array}$} \\
\cline { 2 - 4 } & $2005(\mathrm{n}=4223)$ & $2009(\mathrm{n}=3003)$ & $2014(\mathrm{n}=4914)$ \\
\hline Low FAS & $64.5 \%$ & $72.6 \%$ & $70.6 \%$ \\
\hline Medium/High FAS & $73.0 \%$ & $73.6 \%$ & $76.7 \%$ \\
\hline All & $72.4 \%$ & $73.5 \%$ & $76.3 \%$ \\
\hline Chi-square & $\mathrm{P}<.001 *$ & $\mathrm{P}=0.710$ & $\mathrm{P}=0.022^{*}$ \\
\hline Phi & .050 & .007 & .035 \\
\hline
\end{tabular}

